\title{
Sintomatologia Depressiva e (In)satisfação Escolar
}

\author{
Joana Benevides*, Marina Sousa*, Célia Barreto Carvalho**, Suzana Nunes Caldeira*** \\ *Universidade dos Açores, Portugal, **Universidade dos Açores e Centro de Investigação do Núcleo de Estudos e Intervenção \\ Cognitivo-Comportamental, Portugal, *** Universidade dos Açores - CICS.NOVA.UAçores, Portugal
}

\begin{abstract}
Resumo
A depressão na adolescência tem merecido cada vez mais atenção ocorrendo de forma significativa nesta população. Participaram no estudo 1816 alunos a frequentar o ensino secundário, programa Oportunidades e PROFIJ. Instrumentos: Escala de Ansiedade, Depressão e Stress (EADS-21); Escala de Formas de Auto-criticismo e Auto-tranquilização (FSCRS); Ficha de caracterização sociodemográfica. Resultados: Encontrou-se diferenças estatisticamente significativas entre a sintomatologia depressiva e as formas de auto-criticismo com a satisfação com a escola, com a turma, com os amigos da escola, com os professores e com o desempenho académico.

Palavras-Chave: Perturbações Afetivas, Aprendizagem e Sucesso Escolar, Perturbações Emocionais.
\end{abstract}

\section{Introdução}

A depressão constitui uma patologia com elevada e crescente prevalência na população geral (Bahls, 2002). É atualmente, a nível mundial, a principal causa de incapacitação ocupando o quarto lugar entre as dez principais patologias cabendo a este quadro clínico o segundo lugar nas principais causas de doenças no mundo dentro de 20 anos (OMS, 2002).

Beck, Rush, Shaw e Emery (1979) explicam a depressão, em termos cognitivos, como interpretações sistemáticas acerca das situações, onde o sujeito ativa visões negativas acerca do próprio, do mundo e do futuro. O modelo cognitivo da depressão, sugerido por Beck (1967) assenta em duas conceções básicas de modo a explicar a patologia - tríade cognitiva e distorções cognitivas. Neste sentido a tríade cognitiva respeita à interpretação de experiências de forma negativa, por parte do individuo, tendo-se como inadequado e inapto, bem como uma visão negativa a nível do futuro (Beck, 1967). Por sua vez, as distorções cognitivas conduzem a uma visão absolutista e rígida resultando em erros de interpretação (Beck, 1967) que foram sendo adquiridas ao longo da vida do sujeito (Knapp e Beck, 2008) e que podem ser explicadas por esquemas desenvolvidos desde cedo e mantidos até ao presente (Beck, 1967).

De acordo com o mais recente Manual de Diagnóstico e Estatística das Perturbações Mentais (DSM-5) os sintomas de um episódio depressivo major são os mesmos independentemente da faixa etária. No entanto, a principal característica desta perturbação - a tristeza nas crianças e nos adolescentes pode ser substituída pelo humor irritável. A Perturbação Depressiva Major marca-se por episódios distintos com alterações bem definidas a nível do afeto, da cognição e das funções neurovegetativas (APA, 2014). O humor deprimido, a tristeza, as queixas somáticas, a fadiga, as alterações psicomotoras e de sono, são sintomas da perturbação (APA, 2014). Acresce-se a perda de interesse em atividades que anteriormente eram encaradas como entusiasmantes, alterações de apetite/peso, diminuição da energia, sentimentos de desvalorização e de culpa, dificuldades a nível de pensamento, concentração e tomada de decisão e pensamento recorrente de morte ou ideação suicida ou planos ou tentativas de suicídio (APA, 2014).

A depressão é uma patologia que ocorre de forma significativa na população adolescente (Bahs, 2002), com extensa morbilidade, aguda e crónica (Birmaher \& Brent, 2007) e mortalidade que em muito se deve ao suicídio (APA, 2014). A nível de prevalência a perturbação, a 12 meses, assume o valor de 7\% nos EUA variando consideravelmente de acordo com a faixa etária sendo esta prevalência nas idades compreendidas entre os 18 e 29 anos três vezes superior em relação a 60 anos ou mais (APA, 2014).

A adolescência pode ser considerada como um período de transformação a nível físico, psicológico, afetivo e social. A manifestação desta patologia depende da etapa evolutiva, da personalidade e do ambiente onde o sujeito se encontra inserido podendo ser entendida através das perspetivas - biológica, sociológica e psicológica (Blazer \& Hybels, 2005). Neste seguimento, apontam-se algumas características típicas desta faixa etária associada a este quadro clínico como irritabilidade, instabilidade, momentos de explosão e raiva e ideação suicida (Bahls, 2002). Esta última apresenta índices elevados na população adolescente da Região Autónoma dos Açores (26.3\%) tal como é descrito num estudo realizado por Nunes (2012).

O quadro de depressão clínica mostra-se, assim, gerador de um elevado impacto na vida do individuo comprometendo diversas áreas e contextos em que o individuo se insere.

Quanto aos fatores de risco associados a esta patologia destacam-se os individuais e os ambientais. Neste sentido salienta-se a presença de uma história familiar de depressão, considerada um dos mais potentes fatores de vulnerabilidade à depressão (Dell'Aglio \& Hutz, 2004), sendo também as experiências adversas na infância e acontecimentos geradores de stress apontados como fatores precipitantes para a patologia (APA, 2014). A estes, acrescem-se os fatores temperamentais do indivíduo, como o neuroticismo, os genéticos e os fisiológicos (APA, 2014). Por outro lado, de acordo com 
Abou-Nazel, Fahmy, Younis, Seif el-Din, Fatah e Mokhtar (1991), um fraco desempenho escolar parece encontrar-se, também, associado a um fator de risco para esta patologia na faixa etária dos 11 aos 17 anos, onde a diminuição no rendimento escolar, o humor deprimido, diminuição na capacidade de concentração e fadiga, alterações de sono e apetite e as queixas somáticas são sintomas que poderão servir de alerta em sala de aula (Sukiennik, Segal, Salle, Piltcher, Teruchkin \& Preussler, 2000).

Realça-se, também, a importância do auto-cristicismo, no sentido de se encontrar relacionado com a psicopatologia, nomeadamente com a depressão, como fator da personalidade que conduz à vulnerabilidade desta patologia (Murphy, 2002). O auto-criticismo refere-se a uma forma de auto-condenação e auto-avalição negativa e assume-se como uma dimensão estável da personalidade ligada ao aparecimento da psicopatologia, de dificuldades psicológicas e de um deficitário ajustamento interpessoal (Castilho \& Pinto-Gouveia, 2011).

Desta forma, a interação dos diferentes fatores associam-se a uma maior ou menor probabilidade do surgimento da depressão não descurando a importância e influência dos fatores biológicos/genéticos implicados na etiologia e manutenção deste quadro clínico (Baptista, Baptista \& Dias, 2001).

Partindo deste enquadramento, o presente estudo permitiu relacionar a sintomatologia depressiva em adolescentes com variáveis psicológicas, como o auto-criticismo, e variáveis escolares como, a satisfação com a escola, com a turma, com os amigos e com os professores e o desempenho escolar nas disciplinas de português e de matemática.

\section{Método}

\section{Participantes}

Este estudo considera uma amostra de 1816 alunos do sexo masculino (47.6\%) e feminino (52.4\%), com idades entre os 14 aos 22 anos $(M=16.73$; $D P=1.31)$ que frequentavam o ensino secundário (88.3\%), o programa Oportunidade e PROFIJ (11.7\%). Esta amostra cobriu cerca de $50 \%$ da população adolescente das escolas da ilha de São Miguel.

A nível da variável reprovação escolar, 64.4\% dos alunos do Ensino Secundário nunca reprovou em contraste com alunos do programa Oportunidades e PROFIJ onde apenas $1.4 \%$ dos mesmos nunca foi alvo de reprovação escolar.

\section{Instrumentos}

Ficha de caracterização sociodemográfica.

A operacionalização das variáveis foi concretizada através da ficha de caracterização sociodemográfica que permitiu a recolha de informação respeitante aos participantes a nível do sexo, idade, reprovações, satisfação (com a escola, com a turma, com os amigos da escola e com os professores) e notas das disciplinas de português e matemática.

EADS-21 - Escala de Ansiedade, Depressão e Stress (Lovibond \& Lovibond, 1995; Adaptada à população portuguesa adolescente por Pais-Ribeiro, Honrado e Leal, 2004).

A EADS-21 é constituída por três dimensões: depressão, ansiedade e stress, perfazendo um total de 21 itens. As respostas são dadas numa escala tipo Likert de 4 pontos onde cada item, em forma de afirmação, remete para sintomas emocionais negativos experienciados em relação à última semana, avaliando a sua extensão de acordo com a gravidade ou frequência. Os resultados são determinados pela soma dos valores obtidos em cada uma das dimensões que constituem a escala, 7 itens cada, em que o minino é “ 0 ” e o máximo “21”. Quanto mais elevada a pontuação em cada subescala, mais elevados os estados afetivos mais negativos. Na subescala depressão os valores dominantes do alfa de Cronbach rondam os .60 e nas subescalas ansiedade e stress rondam os .50 (Pais-Ribeiro, Honrado \& Leal, 2004).

FSCRS - Escala das Formas do Auto-criticismo e Auto-tranquilização (FSCRS; Forms of Self-Criticizing and Reassuring Scale Gilbert, Clarke, Hempel, Miles e Irons 2004; Tradução e adaptação de Castilho \& Pinto-Gouveia, 2005)

É uma escala com 22 itens que avalia o modo como as pessoas se auto-criticam/atacam e auto-tranquilizam. É composta por três subescalas - eu inadequado que mede o sentimento de inadequação do eu face a fracassos; eu tranquilizador que indica uma atitude positiva e de compaixão para com o eu e o eu detestado que mede uma resposta mais destrutiva em relação ao eu. As respostas são dadas numa escala de Likert de 5 pontos. Na versão original esta escala apresenta um coeficiente de alfa de Cronbach de .90 para o eu inadequado, .86 para o eu detestado e .86 para o eu tranquilizador (Castilho \& Pinto-Gouveia, 2011).

\section{Procedimento}

Os dados foram recolhidos após alcançadas todas as autorizações necessárias para a realização do estudo. Primeiramente, foi efetuado o levantamento dos dados respeitantes à composição da totalidade das turmas do $10 .^{\circ}, 11 .^{\circ}$ e $12 .^{\circ}$ anos de escolaridade da ilha de São Miguel, e de seguida selecionadas metade das turmas pertencentes a cada ano de escolaridade, sendo esta seleção baseada no método de urna (Poeschl, 2006).

No tratamento estatístico dos dados recorreu-se à versão 21.0 do software de análise estatística: Statistical Packdge for Social Sciences (SPSS).

Procedeu-se a uma análise de regressão linear para avaliar o efeito preditor das variáveis eu detestado, eu inadequado e eu tranquilizador na variável EADS-21 e na dimensão depressão, verificando-se se os modelos são ou não significativos na explicação destes efeitos preditivos. Para se comparar as médias dos grupos, recorreu-se à ANOVA, nomeadamente nas variáveis de satisfação do aluno com a escola, com a turma, com os amigos da escola e com os professores, bem como a nível das notas escolares nas disciplinas de português e matemática. Importa salientar que aquando do cálculo deste teste, foi considerada a igualdade, ou não, das variâncias dos grupos. 


\section{Resultados}

Na amostra estudada os indivíduos apresentam no que diz respeito à EADS-21 uma média mais elevada na dimensão stress $(\mathrm{M}=5.45 ; \mathrm{SD}=4.79)$, seguida da dimensão depressão $(\mathrm{M}=4.50 ; \mathrm{SD}=4.90)$ e, por fim a ansiedade $(\mathrm{M}=3.90 ; \mathrm{SD}=4.25)$. $\mathrm{O}$ score total da EADS-21 apresenta $\mathrm{M}=13.85$ e $\mathrm{SD}=12.96$.

Relativamente à FSCRS verifica-se uma média mais elevada na dimensão eu tranquilizador ( $\mathrm{M}=21.71$; $\mathrm{SD}=6.08)$, seguido do fator eu inadequado $(\mathrm{M}=18.52$, $\mathrm{SD}=7.07)$ e, por último, o eu detestado $(\mathrm{M}=4.28$; $\mathrm{SD}=4.32$ ).

O total da EADS-21, bem como as suas dimensões (ansiedade, stress e depressão) apresentam correlações significativas com as três dimensões da FSCRS (eu inadequado, eu detestado e eu tranquilizador). Assim, há uma correlação moderada com a dimensão eu inadequado $(r=0.45, p=0.000)$ e eu detestado $(r=0,48$, $p=0.000$ ), bem como uma correlação fraca com a dimensão eu tranquilizador $(r=-0,33, p=0.000)$ da FSCRS, com $p<0.01$.

No que diz respeito especificamente à dimensão da depressão da EADS-21 verificam-se correlações significativas com as mesmas dimensões da FSCRS descritas acima. Assim, a dimensão da depressão apresenta correlações moderadas com as dimensões $e u$ detestado ( $r=0.51, p=0.000)$, eu inadequado $(r=0.46$, $p=0.000)$ e eu tranquilizador $(r=-0.40, p=0.000)$, com $p<0,01$.

Para explicar o poder preditor das variáveis de modo a esclarecer a depressão, usou-se a regressão linear. As variáveis significativamente correlacionadas com a EADS-21 total foram incluídas nesta análise: eu detestado, eu inadequado e eu tranquilizador. Os resultados demonstraram que o modelo é significativo e explica 29,1\% da variância total da EADS-21 total (F = 242,669; $p=0.000 ;$ Ra2 $=0.291$ ).

Os coeficientes estandardizados de regressão demonstram que o eu inadequado $(\beta=0.266 ; p=0.000)$, o eu tranquilizador $(\beta=-0.152 ; p=0.000)$ e o eu detestado $(\beta=0.259 ; p=0.000)$ são preditores significativos da EADS-21 total, sendo o eu inadequado o preditor mais forte.

A análise foi também realizada para o fator depressão da EADS-21, sendo que 34,1\% da variância total deste fator é explicada pelas variáveis anteriormente descritas $(\mathrm{F}=305,286 ; \mathrm{p}=0.000 ; \mathrm{Ra} 2=0.341)$. Nesta análise os coeficientes estandardizados de regressão mostraram que o preditor mais forte foi o eu detestado $(\beta=0.272 ; p=$ $0.000)$, seguido do eu inadequado $(\beta=0.255 ; p=0.000)$, e por último o eu tranquilizador $(\beta=-0.215 ; p=0.000)$.

$\mathrm{Na}$ análise de variância entre grupos para as variáveis: satisfação com a escola, com a turma, com os amigos da escola e com os professores considerou-se os seguintes grupos: grupo 1=muito insatisfeito, grupo $2=$ insatisfeito, grupo $3=$ =indiferente, grupo $4=$ satisfeito, grupo 5 =muito satisfeito.

Assim, foi possível verificar no que respeita à variável satisfação com a escola, que existem diferenças estatisticamente significativas entre os grupos no score total da EADS-21 ( $\mathrm{F}=9,014, p=0.000)$, sendo que o grupo 1 possui maior pontuação $(\mathrm{M}=17.79, \mathrm{SD}=14.80)$ do que o grupo $4(\mathrm{M}=12.76, \mathrm{SD}=11.99)$ e $5(\mathrm{M}=11.82$, $\mathrm{SD}=11.85)$ e o grupo 2 possui maior pontuação $(\mathrm{M}=18.35, \mathrm{SD}=15.34)$ do que o grupo $3(\mathrm{M}=14.30$, $\mathrm{SD}=13.43)$, grupo $4(\mathrm{M}=12.76, \mathrm{SD}=11.99)$ e grupo 5 $(\mathrm{M}=11.82, \mathrm{SD}=11.85)$. Quanto à subescala depressão da EADS-21 ( $\mathrm{F}=10.158, \mathrm{p}=0.000)$, verifica-se que $\mathrm{o}$ grupo 1 apresenta maiores pontuações $(M=6.28$, $\mathrm{SD}=5.56)$ que o grupo $4(\mathrm{M}=4.06, \mathrm{SD}=4.47)$ e grupo 5 $(\mathrm{M}=3.63, \mathrm{SD}=4.50)$ e que o grupo 2 apresenta maiores pontuações ( $M=3.65, \mathrm{SD}=4.23)$ que o grupo $3(\mathrm{M}=4.71$, $\mathrm{SD}=5.17)$, grupo $4(\mathrm{M}=4.06, \mathrm{SD}=4.47)$ e $5(\mathrm{M}=3.63$, $\mathrm{SD}=4.50)$.

Quanto à variável satisfação com a turma, verificam-se diferenças estatisticamente significativas entre os grupos para todas as dimensões da EADS-21 e FCSRS. Realça-se o total da EADS-21 ( $\mathrm{F}=9.601, p=$ $0.000)$ e a dimensão depressão $(\mathrm{F}=10.059, p=0.000)$. Para a mesma dimensão verifica-se que o grupo 1 apresenta maiores pontuações $(\mathrm{M}=7.83, \mathrm{SD}=5.92)$ que 0 grupo $4(\mathrm{M}=4.35, \mathrm{SD}=4.79)$ e grupo $5(\mathrm{M}=3.84$, $\mathrm{SD}=4.45)$ e que o grupo 2 apresenta maiores pontuações $(\mathrm{M}=6.01, \mathrm{SD}=5.87)$ que o grupo $5(\mathrm{M}=3.84, \mathrm{SD}=4.45)$ e, por fim, o grupo 3 apresenta maiores pontuações $(\mathrm{M}=5.53, \mathrm{SD}=5.37)$ que o grupo $4(\mathrm{M}=4.35, \mathrm{SD}=4.79)$ e que o grupo $5(\mathrm{M}=3.84, \mathrm{SD}=4.45)$.

Relativamente à variável satisfação com os amigos da escola verificou-se que existem diferenças estatisticamente significativas entre os grupos para a EADS-21 total e as suas dimensões, bem como para todas as dimensões da FSCRS, exceto o eu inadequado. Salientam-se em função do estudo em apreço, a EADS-21 total $(\mathrm{F}=6.270, \mathrm{p}=0.000)$ e a dimensão depressão $(\mathrm{F}=7.638, \mathrm{p}=0.000)$. Para esta última verifica-se que o grupo 3 apresenta maiores pontuações $(\mathrm{M}=6.11, \mathrm{SD}=6.08)$ que o grupo $4(\mathrm{M}=4.51, \mathrm{SD}=4.72)$ e grupo $5(\mathrm{M}=4.08, \mathrm{SD}=4.81)$.

Relativamente à variável satisfação com os professores verificou-se que existem diferenças estatisticamente significativas entre os grupos para a EADS-21 total e suas dimensões e para as dimensões da FSCRS com exceção do eu inadequado. Assim, salientam-se as seguintes: EADS-21 total $(\mathrm{F}=5.856, \mathrm{p}=$ $0.000)$; depressão $(\mathrm{F}=8.229, \mathrm{p}=0.000)$. Para a depressão verifica-se que o grupo 1 e 2 apresentam maiores pontuações $(M=7.66, \quad S D=6.81 ; \quad M=6.27$, $\mathrm{SD}=6.02$, respetivamente) que o grupo $3(\mathrm{M}=4.53$, $\mathrm{SD}=4.59)$, grupo $4(\mathrm{M}=4.19, \mathrm{SD}=5.33)$ e grupo 5 $(\mathrm{M}=4.49, \mathrm{SD}=5.33)$.

Referente à variável nota de português verificou-se que existem, também, diferenças estatisticamente significativas entre os grupos (grupo 1= notas entre os $0 \mathrm{e}$ os 5 valores; grupo 2= notas entre os 6 e os 10 valores; grupo $3=$ notas entre os 11 e os 15 valores; grupo 4= notas entre os 16 e os 20 valores) para a EADS-21 total $(\mathrm{F}=4.135, \mathrm{p}=0.006)$ e suas dimensões depressão $(\mathrm{F}=$ 5.175, $\mathrm{p}=0.001)$; ansiedade $(\mathrm{F}=7.671, \mathrm{p}=0.000)$; stress $(\mathrm{F}=3.260, \mathrm{p}=0.021)$, bem como para o eu detestado $(\mathrm{F}=20.933, \mathrm{p}=0.000)$ e eu tranquilizador $(\mathrm{F}$ $=4.678, \mathrm{p}=0.003)$ da FSCRC sendo que para o eu inadequado não existem diferenças estatisticamente significativas entre os grupos $(F=1.471, p=0.222)$. Na 
dimensão depressão verificam-se pontuações mais elevadas no grupo $1 \quad(\mathrm{M}=4.95, \quad \mathrm{SD}=5.24)$, comparativamente ao grupo $3(\mathrm{M}=3.99, \mathrm{SD}=4.38)$.

Por fim, no que respeita à variável nota de matemática, verificou-se que existem diferenças estatisticamente significativas entre os grupos, acima descritos, para a EADS-21 total $(\mathrm{F}=3.804, \mathrm{p}=0.010)$, sendo que o grupo 1 apresenta pontuações mais elevadas ( $\mathrm{M}=14.88$ $\mathrm{SD}=13.86)$, quando comparado com o grupo $3(\mathrm{M}=12.26$ $\mathrm{SD}=11.04)$. Na dimensão da depressão verificou-se que também existem diferenças estatisticamente significativas entre os grupos $(\mathrm{F}=4.817, p=0.002)$, sendo que o grupo 1 apresenta pontuações mais elevadas $(\mathrm{M}=4.91 \mathrm{SD}=5.21)$, quando comparado com o grupo 3 $(\mathrm{M}=3.86 \mathrm{SD}=4.26)$.

\section{Discussão}

Os resultados deste estudo permitiram averiguar a relação entre a sintomatologia depressiva, o auto-criticismo e a satisfação com a escola, com os amigos da escola, com a turma, com os professores e com as notas de português e matemática numa população adolescente.

Deste modo, observou-se que a EADS-21, e as suas subescalas, apresentaram uma correlação significativa com a FSCRS sugerindo uma relação com a forma como as pessoas se auto-criticam e auto-tranquilizam. Este resultado vai ao encontro da literatura nomeadamente ao observado no estudo de Luthar e Blatt (1995) onde os alunos com autocritica elevada apresentaram, na investigação, maior sintomatologia depressiva associada a dificuldades escolares e funcionamento interpessoal deficitário. No estudo de Fröjd, Nissinen, Pelkonen, Marttunen, Koivisto e Kaltiala-Heino (2008) a depressão foi associada a dificuldades de concentração, relações sociais, desempenho escolar, leitura e escrita, e, neste seguimento, os autores afirmam a premência em rastrear a depressão em alunos com dificuldades no desempenho escolar. Níveis mais elevados de depressão foram associados a um desempenho escolar inferior no estudo de Owens, Stevenson, Hadwin e Norgate 2012), ao que Fergusson e Woodward (2002) acrescentam que a depressão tende a ser preditora de dificuldades a nível escolar.

Foram também encontradas diferenças estatisticamente significativas entre a subescala da EADS-21 depressão e as variáveis satisfação com a escola, com a turma, com os amigos da escola e com os professores. De acordo com os resultados descritos, os alunos mais satisfeitos com estas variáveis tenderam a ter valores mais baixos na dimensão depressão. Krause, Liang e Yatomi (1989 citado por Baptista, Baptista \& Dias, 2001) defendem que os sintomas depressivos podem sofrer influência da insatisfação que o sujeito sente com o seu grupo social, onde os relacionamentos construtivos com a família e amigos poderão prevenir um quadro depressivo (Rubin, Rubenstein, Stechler, Heeren, Halton, Housman e Linda-Kasten, 1992 citado por Batista, Batista \& Dias, 2001). Por outro lado, segundo Carson \& Bittner, 1994 citado por, Dell'Aglio e Hutz (2004), eventos stressantes em contexto escolar, nomeadamente conflitos com os companheiros e professores, podem, eventualmente, traduzir-se em fobias, queixas somáticas e episódios depressivos. No estudo levado a cabo por Barth, Hofmann e Schori (2014), com uma amostra de 10740 participantes com idades compreendidas entre os 18 e 25 anos, observou-se que as pessoas indicadas com depressão associavam-se uma perda substancial na satisfação com a escola. Esta relação poderá levar a um prognóstico global menos positivo da qualidade de vida tendo em conta as limitações a nível de saúde mental e de recursos com que o sujeito se depara (Barth, Hofmann e Schori, 2014).

Pela exploração da associação entre a subescala, depressão e a nota de português e de matemática foi possível verificar que os alunos cujas notas residiam entre os 0 e os 5 valores tendiam a obter valores mais elevados a nível da sintomatologia depressiva. Pérez e Urquijo (2001) estudaram a associação entre a sintomatologia depressiva em adolescentes e o desempenho escolar encontrando associações negativas estatisticamente significativas entre total do teste Children's Depression Scale (CDI) e o desempenho nas disciplinas de Matemática e Língua (Espanhol) sugerindo que à medida que aumentam os níveis de depressão diminuem as pontuações do desempenho escolar. De modo mais específico, no estudo de Bandim, Roazzi e Doménech (1998 citado por Dell'Aglio \& Hutz) sobre o rendimento escolar em crianças dos 9 aos 12 anos com sintomatologia depressiva, verificou-se um prejuízo significativo no desempenho escolar em todas as disciplinas, de onde se realça a Língua Portuguesa, quando comparadas com crianças sem sintomatologia depressiva. No estudo levado a cabo por Cruvinel e Boruchovitch (2004) também se verificou uma associação estatisticamente significativa entre os sintomas depressivos e a nota de Matemática. Neste sentido o resultado pode ser interpretado à luz do referido por Dell’Aglio e Hutz (2004), onde o fraco desempenho escolar tende a que o sujeito apresente índices maiores de depressão uma vez que não se sente capaz de lidar com os pedidos impostos pelo meio. Por outro lado observa-se uma associação estatisticamente significativa que pressupõe que adolescentes com notas mais fracas em Português tenham níveis mais elevados na dimensão eu detestado. De acordo com Brumback, Jackoway e Weinberg (1980), um fraco rendimento escolar poderá ocorrer como consequência da sintomatologia depressiva, quer em função da falta de interesse e motivação do aluno, quer pelos sentimentos de autodesvalorização inerentes à patologia.

Considerando os resultados deste estudo e a alta prevalência da depressão na adolescência torna-se imperativo a avaliação, a construção e a implementação de programas que permitam uma intervenção precoce eficaz para a redução dos sintomas depressivos promovendo a saúde mental. Em contexto escolar é fundamental que os educadores/professores se mantenham atentos a sinais nos adolescentes que possa pressupor sofrimento psíquico, nomeadamente a sintomatologia depressiva, a fim de evitar repercussões como é o caso do insucesso ou mesmo do abandono escolar. Assim, fazendo uma análise custo-benefício, com a necessidade de remediação a vários níveis, a 
ausência de uma intervenção precoce implicará caras intervenções terciarias, tanto a nível educativo quanto hospitalar.

\section{Referências}

Abou-Nazel, M.W., Fahmy, S.I., Younis, I.A., Seif el-Din, A.G., Fatah, M.A., Mokhtar, S. \& Ayoub, A.L. (1991). A Study of Depression among Alexandria Preparatory School Adolescents. J Egypt Public Health Assoc, 66(5-6), 649-674. http://www.ncbi.nlm.nih.gov/pubmed/1797970

American Psychiatric Association (2014). DSM-5: Manual de Diagnóstico e Estatística das Perturbações Mentais. $5^{\text {a }}$ Edição. Lisboa: Climepsi

Bahls, S. C. (2002). Aspectos clínicos da depressão em crianças e adolescentes. Jornal de Pediatria, 78(5), 359-366. doi: 10.1590/S0021-75572002000500004

Baptista, M. N., Baptista, A. S. D., \& Dias, R. R. (2001). Estrutura e suporte familiar como fatores de risco na depressão de adolescentes. Psicologia: Ciência $e$ Profissão, 21(2), 52-61. doi: 10.1590/S1414-98932001000200007

Barth, J., Hofmann, K., \& Schori, D. (2014). Depression in early adulthood: Prevalence and psychosocial correlates among young Swiss men. Swiss Med Wkly, 144, w13945. doi:10.4414/smw.2014.13945

Beck, A. T. (1967). Depression: Clinical, experimental, and theoretical aspects. New York: Harper \& Row.

Beck, A. T., Rush, A. J., Shaw, B. F. \& Emery, G. (1979). Cognitive Therapy of Depression. New York: The Guilford Press.

Birmaher, B. \& Brent, D. (2007). Practice parameter for the assessment and treatment of children and adolescents with depressive disorders. Journal of the American Academy of Child \& Adolescent Psychiatry, 46(11), 1503-1526. doi: 10.1097/chi.0b013e318145ae1c

Blazer, D. G., \& Hybels, C. F. (2005). Origins of depression in later life. Psychological medicine, 35(09), 1241-1252. doi: 10.1017/S0033291705004411

Brumback, R.A, Jackoway, M.K. \& Weinberg, W.A. (1980). Relation of Intelligence to childhood depression in children referred to Educational Diagnostic Center. Perceptual and Motor Skills. 50, 11-17. doi: 10.2466/pms.1980.50.1.11

Castilho, P., \& Gouveia, J. P. (2011). Auto-Criticismo: estudo de validação da versão portuguesa da Escala das Formas do Auto-Criticismo e Auto-Tranquilização (FSCRS) e da Escala das Funções do Auto-Criticismo e Auto-Ataque (FSCS). Psychologica, (54), 63-86. http://iduc.uc.pt/index.php/psychologica/article/viewFil e/1101/549

Cruvinel, M., \& Boruchovitch, E. (2004). Sintomas depressivos, estratégias de aprendizagem e rendimento escolar de alunos do ensino fundamental. Psicologia em estudo, 9(3), 369-378. doi: 10.1590/S1413-73722004000300005

Dell’Aglio, D. D., \& Hutz, C. S. (2004). Depressão e desempenho escolar em crianças e adolescentes institucionalizados. Psicologia: Reflexão e Crítica,
17(3), 341-350. doi: 10.1590/S0102-79722004000300008

Fergusson, D. M., \& Woodward, L. J. (2002). Mental health, educational, and social role outcomes of adolescents with depression. Archives of general psychiatry, 59(3), 225-231. doi: 10.1001/archpsyc.59.3.225

Fröjd, S. A., Nissinen, E. S., Pelkonen, M. U., Marttunen, M. J., Koivisto, A. M., \& Kaltiala-Heino, R. (2008). Depression and school performance in middle adolescent boys and girls. Journal of adolescence, 31(4), 485-498. doi: 10.1016/j.adolescence.2007.08.006

Knapp, P., \& Beck, A. T. (2008). Fundamentos, modelos conceituais, aplicações e pesquisa da terapia cognitiva. Revista Brasileira de Psiquiatria, 30(Supl II), S54-64. doi: 10.1590/S1516-44462008000600002

Luthar, S.; Blatt, S. J. (1995). Differential vulnerability of dependency and self-criticism among disadvantaged teenagers. Journal of Research on Adolescence, 5(4), 431-449. doi: 10.1207/s15327795jra0504_3

Murphy, J.M., Nierenberg, A.A., Monson, R.R., Laird, N.M., Sobol, A.M. \& Leighton, A.H. (2002). Self-disparagement as a feature and forerunner of depression: Findings from the Stirling County study. Comprehensive Psychiatry, 43, 13-21. doi: 10.1053/comp.2002.29842

Nunes, C.P.S. (2012), Auto-dano e ideação suicida na população adolescente Aferição do Questionário de Impulso, Auto-dano e Ideação suicida na Adolescência (QIAIS-A). Dissertação de mestrado. Universidade dos Açores, Ponta Delgada.

OMS, Organização Mundial da Saúde (2002), Relatório Mundial de Saúde. Saúde Mental, Nova Concepção, Nova Esperança, Lisboa, Direção-Geral da Saúde / OMS. http://www.who.int/whr/2001/en/whr01_po.pdf

Owens, M., Stevenson, J., Hadwin, J. A., \& Norgate, R. (2012). Anxiety and depression in academic performance: An exploration of the mediating factors of worry and working memory. School Psychology International, 33(4), 433-449. doi: 10.1177/0143034311427433

Pérez, M. V., \& Urquijo, S. (2001). Depresión en adolescentes. Relaciones com el desempeñho académico. Psicologia Escolar e Educacional, 5(1), 49-58. doi: 10.1590/S1413-85572001000100006

Poeschl, G. (2006). Análise de dados na investigação em psicologia: Teoria e prática. Edições Almedina. Coimbra.

Powell, V. B., Abreu, N., de Oliveira, I. R., \& Sudak,D. (2008). Terapia cognitivo-comportamental da depressão. Revista Brasileira de Psiquiatria, 30(Supl II), S73-80. http://www.scielo.br/pdf/rbp/v30s2/a04v30s2.p df

Ribeiro, J. L. P., Honrado, A. A. J. D., \& Leal, I. P. (2004). Contribuição para o estudo da adaptação portuguesa das escalas de ansiedade, depressão e stress (EADS) de 21 itens de Lovibond e Lovibond. Psicologia, Saúde \& Doenças, 5(2), 229-239. http://sigarra.up.pt/fpceup/pt/publs_pesquisa.s how_publ_file?pct_gdoc_id=4875 
Sukiennik, P. B., Segal, J., Salle, E., Piltcher, R. B., Teruchkin, B., \& Preussler, C. M. (2000). Implicações da depressäo e do risco de suicídio na escola durante a adolescência. Adolescencia Latinoamericana, 2(1), 36-44. http://ral-adolec.bvs.br/pdf/ral/v2n1/p07v2n1.pdf 